\title{
Clinical Significance of Subcellular Localization of Maspin in Patients with Pathological Stage IA Lung Adenocarcinoma
}

\author{
TAKASHI OHNO ${ }^{1,2}$, YASUAKI KUBOUCHI ${ }^{1,2}$, MAKOTO WAKAHARA $^{1,2}$, \\ KANAE NOSAKA ${ }^{1}$, TOMOHIKO SAKABE ${ }^{1}$, TOMOHIRO HARUKI ${ }^{2}$, KEN MIWA $^{2}$, \\ YUJI TANIGUCHI ${ }^{2}$, HIROSHIGE NAKAMURA $^{2}$ and YOSHIHISA UMEKITA ${ }^{1}$ \\ ${ }^{1}$ Division of Organ Pathology, Department of Pathology and ${ }^{2}$ Division of General Thoracic Surgery, \\ Department of Surgery, Faculty of Medicine, Tottori University, Tottori, Japan
}

\begin{abstract}
Background/Aim: Maspin is a tumor-suppressor protein and its prognostic value in lung adenocarcinoma has been reported. However, little is known about the clinical impact of subcellular localization of maspin in early-stage lung adenocarcinoma. We aimed to evaluate the clinical significance of subcellular localization of maspin in patients with pathological stage (p-stage) IA lung adenocarcinoma categorized by the new eighth edition TNM classification. Patients and Methods: We immunohistochemically analyzed 181 tissue samples from p-stage IAl ( $n=37)$, IA2 $(n=92)$ and IA3 ( $n=52)$ lung adenocarcinomas using antibody for maspin. Results: The 181 cases fell into five predominant subtypes: lepidic ( $n=32)$, acinar $(n=97)$, papillary $(n=30)$, solid $(n=20)$ and micropapillary $(n=2)$. The frequencies of maspin staining were: cytoplasmic-only in 24.9\%; pancellular (nuclear and cytoplasmic) in 8.8\%; nuclear-only in 0.6\%; no staining in 65.7\%. Cytoplasmic-only staining significantly correlated with high pathological T-classification $(p=0.039)$, lymphatic invasion $(p=0.002)$ and poorer tumor differentiation ( $p=0.002)$. The patients were followed-up for 12-151 months (median=74 months), and the cytoplasmic-only staining significantly correlated with shorter disease-free survival (DFS) ( $p=0.034)$ and disease-specific survival (DSS) $(p=0.036)$ by log-rank tests. In Cox's multivariate analysis, lymphatic invasion had the most significant effect on shorter DFS and DSS. Conclusion: The expression of maspin in the cytoplasm alone could be useful for predicting unfavorable prognoses in patients with p-stage IA lung adenocarcinoma.
\end{abstract}

Correspondence to: Professor Yoshihisa Umekita, Division of Organ Pathology, Department of Pathology, Faculty of Medicine, Tottori University, 86 Nishicho, Yonago, Tottori 683-8503, Japan. Tel: +81 859386053, Fax: +81 859386050, e-mail: yume@med.tottori-u.ac.jp

Key Words: Maspin, adenocarcinoma, immunohistochemistry.
Adenocarcinoma is the most frequent histological subtype of lung cancer accounting for $>40 \%$ of all lung cancers (1). With the development of imaging technology, smaller and earlierstage lung adenocarcinomas are now being detected. However, the 5-year recurrence rate of adenocarcinoma was reported to range from $16 \%$ to $20 \%$ even in patients with pathological stage (p-stage) I disease (2-4). The eighth edition of the tumor-node-metastasis (TNM) classification of lung cancer was recently published, and stage IA was divided into stages IA1, IA2 and IA 3 according to the greatest dimension of the invasive component (5). The 5-year survival rates of patients with stage IA1, IA2 and IA 3 cancer were reported to be $92 \%, 83 \%$ and $77 \%$, respectively (6).

Maspin, a non-inhibitory member of the serine protease inhibitor (serpin) superfamily, was detected originally as a tumor-suppressor protein expressed in normal breast epithelial cells but whose expression is reduced in or absent from breast carcinoma (7). Maspin has been shown to inhibit both tumor growth and metastasis in multiple animal models and cancer cell lines, and has shown pro-apoptotic, antimetastatic and anti-angiogenic properties, exerting an inhibitory effect on cancer cell motility, invasiveness and metastasis ability (8). Although the exact biochemical pathways leading to these biological endpoints are incompletely characterized, several studies have reported an association between maspin expression and clinicopathological factors in many types of cancer, including lung cancer. However, there are conflicting results regarding whether maspin expression is a favorable or unfavorable indicator in patients with lung cancer (9-15). We also reported that the cytoplasmic-only expression of maspin was a poor prognostic indicator in patients with lung adenocarcinoma measuring $<3 \mathrm{~cm}$ (13). However, that study included adenocarcinoma in situ (AIS) and minimally invasive adenocarcinoma (MIA), for which the 5-year disease-free survival (DFS) rate and 5-year disease-specific survival (DSS) rate are both 100\% (16). To our knowledge, 
there is no report validating the clinical impact of maspin expression in p-stage IA lung adenocarcinoma as categorized by the new TNM classification. The aim of our study was to clarify the prognostic value of maspin expression with focus on its subcellular localization in patients with p-stage IA lung adenocarcinoma.

\section{Patients and Methods}

Patients and tumor specimens. From January 2005 to December 2012 at the Tottori University Hospital (Tottori, Japan), 229 consecutive patients underwent curative surgical resection of lung cancer diagnosed as p-stage IA lung adenocarcinoma according to the eighth edition of the TNM classification (5). Eight cases were excluded for the following reasons: in two cases, a history of neoadjuvant chemotherapy was noted; in three cases, there was a history of radiation therapy; and in the remaining three cases, death occurred within 30 days after surgery (due to pulmonary embolism, adult respiratory distress syndrome and acute renal failure). Nine cases of invasive mucinous adenocarcinoma and 31 cases of MIA were also excluded. Thus, 181 cases with p-stage IA (IA1 in 37 cases, IA 2 in 92 and IA3 in 52) were included in this study. The median follow-up time was 74 months (range=12-151 months). The patients' clinicopathological data were obtained from their hospital medical records.

Histopathological evaluation. The pathological diagnosis was performed using hematoxylin and eosin (H\&E)-stained sections according to the criteria of the current World Health Organization (WHO) classification of lung cancer (1), and all H\&E slides were reviewed by T.O. and Y.U. without knowledge of any of the clinical data. The other histopathological factors, such as tumor differentiation and tumor size were diagnosed histopathologically. The size of the invasive component was evaluated by Elastica van Gieson staining. Lymphatic invasion was evaluated by immunohistochemistry using an antibody for podoplanin. The tumor stage was determined based on the eighth edition of the TNM classification of lung cancer (5). All cases were classified into five predominant subtypes, namely lepidic, acinar, papillary, solid or micropapillary, according to the WHO classification of lung adenocarcinoma (1). Written informed consent for their data to be used was obtained from all patients, and the present study was approved by the Ethics Committee of the Faculty of Medicine, Tottori University (approval no.: 1706A059; June 14, 2017).

Immunohistochemistry. All specimens were fixed in $10 \%$ neutrally buffered formalin and embedded in paraffin. After sections $(4-\mu \mathrm{m}$ thick) were deparaffinized and endogenous peroxidase activity was blocked, they were pretreated in citrate buffer $(0.01 \mathrm{M}, \mathrm{pH} 6.0)$ in a microwave oven for $15 \mathrm{~min}$. We then performed the immunohistochemical examination using a monoclonal antibody to human maspin (clone EAW24, diluted 1:150; Leica Biosystems, Newcastle upon Tyne, UK) as described elsewhere (15).

Evaluation of immunohistochemical findings. We assessed the cells as being positive for maspin expression at any location when strong staining was identified. Strong staining was defined as a staining intensity equal to that of the nuclei of basal cells of the bronchus, and that staining intensity served as an internal positive control
Table I. Clinicopathological characteristics of 181 patients with pathological stage IA lung adenocarcinoma.

\begin{tabular}{lr}
\hline Variable & Value \\
\hline Age (mean \pm SD, years) & $69.7 \pm 9.5$ \\
Gender, $\mathrm{n}(\%)$ & \\
Male & $87(48.1)$ \\
Female & $94(51.9)$ \\
Smoking history, $\mathrm{n}(\%)$ & \\
Ever smoker & $82(45.3)$ \\
Never smoker & $99(54.7)$ \\
Surgical procedure, $\mathrm{n}(\%)$ & \\
Segmentectomy & $28(15.5)$ \\
Lobectomy & $153(84.5)$ \\
Tumor size (mean \pm SD), mm & $21.9 \pm 7.9$ \\
Size of invasive component $($ mean $\pm S D)$, mm & $16.9 \pm 6.7$ \\
Pathological tumor status, $\mathrm{n}(\%)$ & \\
pT1a & $37(20.4)$ \\
pT1b & $92(50.8)$ \\
pT1c & $52(28.7)$ \\
Lymphatic invasion, $\mathrm{n}(\%)$ & $39(21.5)$ \\
Present & $142(78.5)$ \\
Absent & \\
Tumor differentiation, $\mathrm{n}(\%)$ & $32(17.7)$ \\
Well & $127(70.2)$ \\
Moderate & $22(12.2)$ \\
Poor & $32(17.7)$ \\
Histological subtype, $\mathrm{n}(\%)$ & $97(53.6)$ \\
Lepidic & $30(16.6)$ \\
Acinar & $20(11.0)$ \\
Papillary & $2(1.1)$ \\
Solid & \\
Micropapillary & \\
\hline & \\
\hline
\end{tabular}

$\mathrm{SD}$, Standard deviation.

when present (17). Tumors with $>10 \%$ positive cells were considered maspin-positive. Alveolar epithelial cells served as an internal negative control (17). All slides were evaluated by T.O. and Y.U., who were blinded to the patient clinicopathological data.

Statistical analysis. All statistical analyses were performed using SPSS ver. 23 software (IBM SPSS Statistics, IBM, Armonk, NY, USA). We evaluated the association between maspin status and clinicopathological factors by performing non-parametric tests. The Chi-square test was used when there were two categorical variables of interest and the Kruskal-Wallis test was used when there were three or more variables. For the survival analysis, we used two different endpoints to calculate the DFS and DSS rates: cancer relapse (local recurrence or distant recurrence) and cancer-related death, respectively. DFS was defined as the period from the date of initial surgery to the date of clinical or pathological cancer relapse. DSS was defined as the period from the date of initial surgery to the date of cancer-related death. The cases of the patients who died from a cause unrelated to their lung cancer or for whom the cause of death was not entirely clear were censored for the purposes of survival analyses. Survival curves were computed according to the Kaplan-Meier method, and differences in the DFS and DSS were analyzed using the log-rank test. We used the Cox hazard regression 

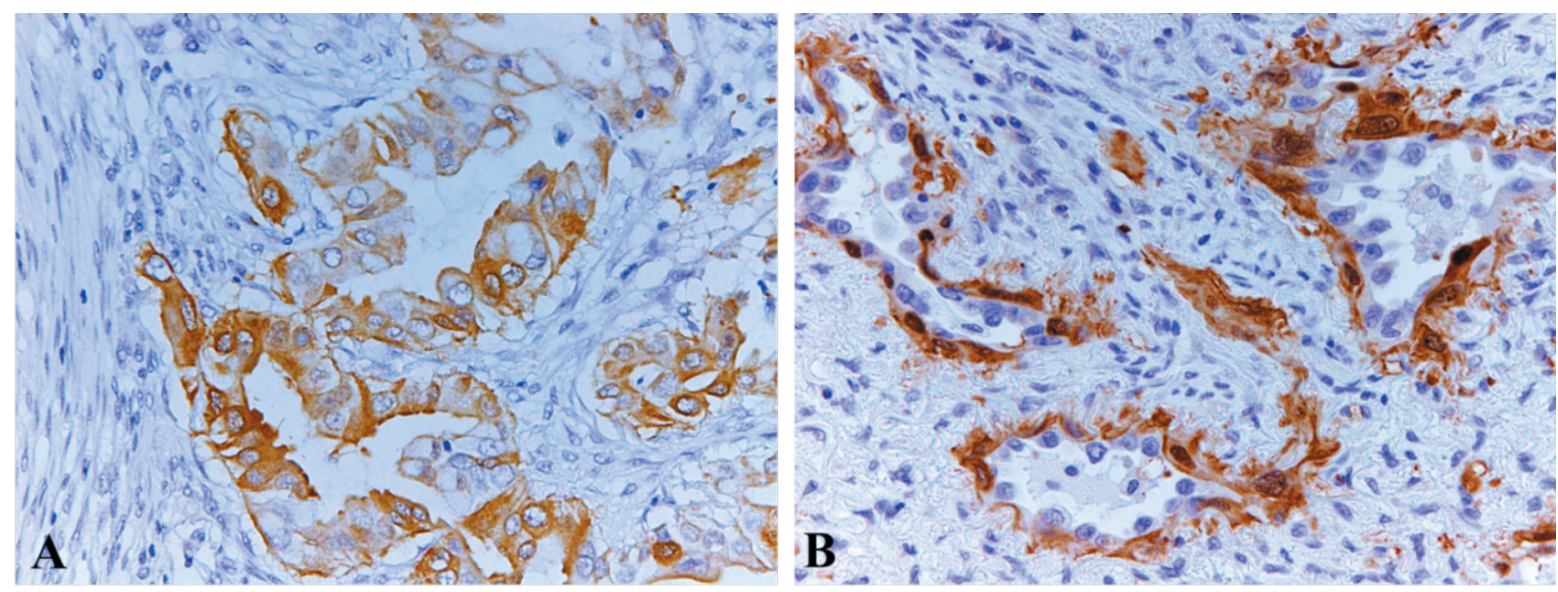

Figure 1. Immunohistochemical staining of maspin in lung adenocarcinoma. A: Strong cytoplasmic-only expression of maspin. B: Pancellular (nuclear and cytoplasmic) expression of maspin.

model to evaluate the effects of various factors on the DFS and DSS in order to determine the independent prognostic value of maspin status. All tests of significance were two-sided, and $p$-values of less than 0.05 were considered significant in all tests.

\section{Results}

Immunohistochemistry. Representative immunohistochemical staining patterns of maspin expression are shown in Figure 1. In normal lung tissue, the nuclei of basal cells of the bronchus stained strongly, whereas alveolar epithelial cells were not stained. The subcellular localization of maspin expression was classified into four categories: cytoplasmiconly in 45 cases $(24.9 \%)$, pancellular (combined nuclear and cytoplasmic) in 16 cases $(8.8 \%)$, nuclear-only in one case $(0.6 \%)$ and no staining in 119 cases $(65.7 \%)$. No cases of membrane-only staining were observed. Strong staining intensity was observed in 62 cases (cytoplasmic-only in 45 cases, pancellular in 16 cases and nuclear-only in one case), weak staining in 18 cases and no staining in 101 cases.

Clinicopathological characteristics and correlation with maspin expression. The clinicopathological characteristics of the 181 patients with adenocarcinoma are summarized in Table I. A total of 181 cases with invasive adenocarcinoma were classified into five subtypes: lepidic-predominant in 32 patients $(17.7 \%)$, acinar-predominant in 97 patients $(53.6 \%)$, papillary-predominant in 30 patients $(16.6 \%)$, solidpredominant in 20 patients $(11.0 \%)$ and micropapillarypredominant in two patients $(1.1 \%)$. The correlations between the clinicopathological characteristics and maspin immunostaining are summarized in Table II. The pancellular expression of maspin was not correlated with any clinicopathological factor, whereas cytoplasmic-only expression of maspin was significantly correlated with a greater frequency of high pathological T-classification $(p=0.039)$, lymphatic invasion $(p=0.002)$, and poor tumor differentiation $(p=0.002)$.

Survival analysis. Fourteen and 13 patients experienced locoregional and distant recurrence, respectively. Eleven patients died of lung cancer progression and 23 died of other causes, including other cancer-related death, pneumonia, interstitial pneumonia and cerebral hemorrhage. The survival curves for the patients are shown in Figures 2 and 3. The 5year DFS rates of the group with cytoplasmic-only maspin staining versus the other categories were $67.9 \%$ versus $81.0 \%$, and the corresponding 5-year DSS rates were $92.5 \%$ versus $97.9 \%$, respectively. The log-rank test showed that the group with cytoplasmic-only staining had significantly shorter DFS and DSS values compared to the other groups ( $p=0.034$ and $p=0.036$, respectively). The 5 -year DFS rates of the groups with pancellular and nuclear-only staining combined and of the no-staining group were $75.0 \%$ and $81.5 \%$, respectively, and the corresponding 5-year DSS rates were $93.3 \%$ and $98.8 \%$. The log-rank test showed that the cytoplasmic-only staining group had significantly shorter DFS and DSS values compared to the no-staining group ( $p=0.041$ and $p=0.037$, respectively) (Figure 3). Univariate analyses showed significant correlations between shorter DFS and cytoplasmic-only staining $(p=0.037)$, higher age $(p=0.029)$, higher pathological T-classification $(p=0.029)$, and the presence of lymphatic invasion $(p<0.001)$ (Table III). Cytoplasmic-only staining $(p=0.048)$ and the presence of lymphatic invasion $(p=0.008)$ were also significantly correlated with shorter DSS. According to the multivariate analysis using the Cox hazard regression model, the presence of lymphatic invasion had the most significant effect on DFS $(p<0.001)$ and DSS $(p=0.008)$ (Table IV). 
Table II. Association between subcellular localization of maspin and clinicopathological characteristics.

\begin{tabular}{|c|c|c|c|c|c|c|c|}
\hline \multirow[t]{2}{*}{ Factor } & \multirow[b]{2}{*}{$\begin{array}{c}\text { Total } \\
(\mathrm{N}=181)\end{array}$} & \multicolumn{3}{|c|}{ Cytoplasmic-only expression, $\mathrm{n}$} & \multicolumn{3}{|c|}{ Pancellular expression, $\mathrm{n}$} \\
\hline & & $\begin{array}{c}\text { Positive } \\
(\mathrm{N}=45)\end{array}$ & $\begin{array}{l}\text { Negative } \\
(\mathrm{N}=136)\end{array}$ & $p$-Value & $\begin{array}{c}\text { Positive } \\
(\mathrm{N}=16)\end{array}$ & $\begin{array}{l}\text { Negative } \\
(\mathrm{N}=165)\end{array}$ & $p$-Value \\
\hline \multicolumn{8}{|l|}{ Age } \\
\hline$<70$ Years & 75 & 15 & 60 & \multirow[t]{2}{*}{0.203} & 8 & 67 & \multirow[t]{2}{*}{0.466} \\
\hline$\geq 70$ Years & 106 & 30 & 76 & & 8 & 98 & \\
\hline \multicolumn{8}{|l|}{ Gender } \\
\hline Male & 87 & 25 & 62 & \multirow[t]{2}{*}{0.246} & 5 & 82 & \multirow[t]{2}{*}{0.159} \\
\hline Female & 94 & 20 & 74 & & 11 & 83 & \\
\hline \multicolumn{8}{|l|}{ Smoking history } \\
\hline Ever smoker & 82 & 26 & 56 & \multirow[t]{2}{*}{0.052} & 5 & 77 & \multirow[t]{2}{*}{0.237} \\
\hline Never smoker & 99 & 19 & 80 & & 11 & 88 & \\
\hline \multicolumn{8}{|l|}{ Surgical procedure } \\
\hline Segmentectomy & 28 & 9 & 19 & \multirow[t]{2}{*}{0.332} & 2 & 26 & \multirow[t]{2}{*}{0.731} \\
\hline Lobectomy & 153 & 36 & 117 & & 14 & 139 & \\
\hline \multicolumn{8}{|c|}{ Pathological tumor status } \\
\hline pT1a & 37 & 5 & 32 & \multirow[t]{3}{*}{0.039} & 4 & 33 & \multirow[t]{3}{*}{0.325} \\
\hline pT1b & 92 & 21 & 71 & & 10 & 82 & \\
\hline pT1c & 52 & 19 & 33 & & 2 & 50 & \\
\hline \multicolumn{8}{|l|}{ Lymphatic invasion } \\
\hline Present & 39 & 17 & 22 & \multirow[t]{2}{*}{0.002} & 3 & 36 & \multirow[t]{2}{*}{0.776} \\
\hline Absent & 142 & 28 & 114 & & 13 & 129 & \\
\hline \multicolumn{8}{|c|}{ Tumor differentiation } \\
\hline Well & 32 & 5 & 27 & \multirow[t]{3}{*}{0.002} & 5 & 27 & \multirow[t]{3}{*}{0.139} \\
\hline Moderate & 127 & 28 & 99 & & 11 & 116 & \\
\hline Poor & 22 & 12 & 10 & & 0 & 22 & \\
\hline \multicolumn{8}{|c|}{ Histological subtype } \\
\hline Lepidic & 32 & 5 & 27 & & 5 & 27 & \\
\hline Acinar & 97 & 20 & 77 & & 7 & 90 & \\
\hline Papillary & 30 & 8 & 22 & & 4 & 26 & \\
\hline Solid & 20 & 11 & 9 & & 0 & 20 & \\
\hline Micropapillary & 2 & 1 & 1 & & 0 & 2 & \\
\hline
\end{tabular}

\section{Discussion}

Several studies investigated the prognostic value of maspin expression in patients with non-small cell lung carcinoma (NSCLC) (9-12) or adenocarcinoma only (13-15), but there are conflicting results regarding whether maspin expression is a favorable or unfavorable indicator. Hirai et al. reported that cytoplasmic maspin expression was a significant poor prognostic factor in 132 patients with NSCLC including 94 adenocarcinomas (9). Lonardo et al. reported that the nuclear-only expression of maspin may be useful to stratify subtypes of lung adenocarcinoma with favorable clinicopathological features (13). By contrast, the authors of the largest series (352 NSCLCs) reported that the cytoplasmic or nuclear expression of maspin was not correlated with tumor-specific survival (12). Factors contributing to the potential complexity may include the differences in the study populations (especially TNM stage), the histological types investigated, the antibodies used, criteria for positivity, including cut-offs, and whether subcellular localization was examined.

To our knowledge, only two prior studies have investigated the prognostic value of maspin expression in patients with early-stage lung adenocarcinoma (14, 15). Frey et al. reported that the nuclear-only expression of maspin was the only predictor of improved survival in 46 patients with stage I lung adenocarcinomas (14). We also demonstrated that the cytoplasmic-only expression of maspin was an independent poor prognostic indicator in 110 patients with lung adenocarcinoma measuring $<3 \mathrm{~cm}$ (15). However, our previous study included AIS and MIA cases, for which the 5-year DFS rate and 5-year DSS rate are both 100\% (16), and it also included patients with stage IB $(n=8)$, IIA $(n=3)$, IIB $(n=2)$ and IIIA $(n=9)$ adenocarcinomas. In the present study, we thus attempted to more strictly stratify patients with early-stage lung adenocarcinoma by using the new edition of the TNM classification of lung cancer. The present patient series thus included patients with p-stage IA lung 

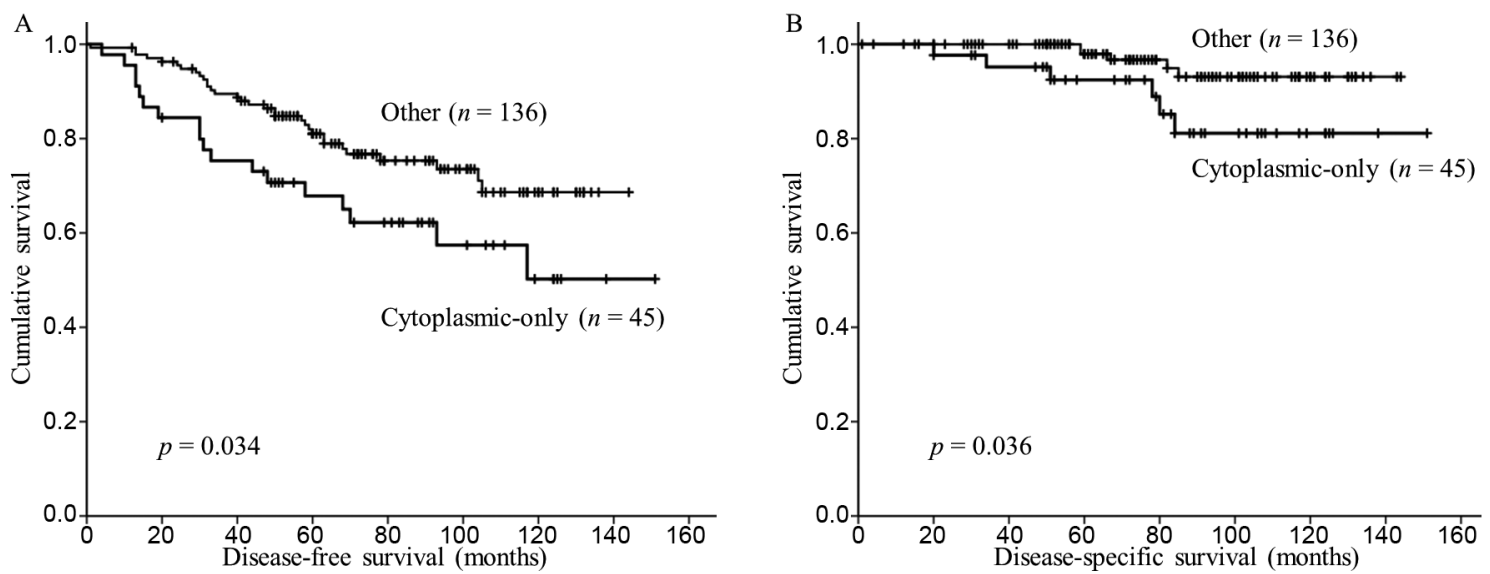

Figure 2. Kaplan-Meier survival curves for disease-free (A) and disease-specific (B) survival of 181 patients according to maspin expression status (cytoplasmic-only staining versus all other categories).

A

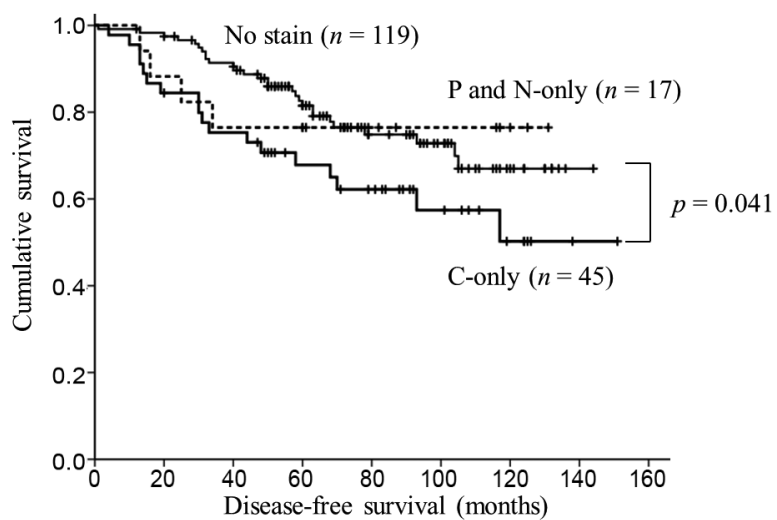

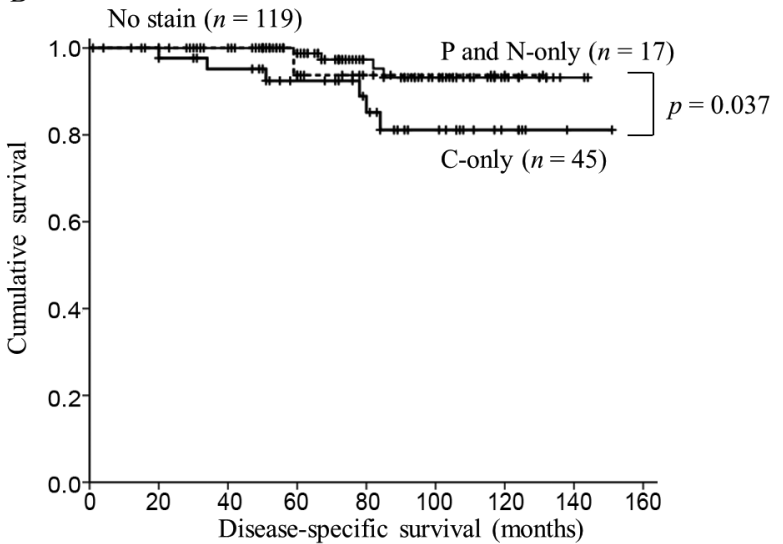

Figure 3. Kaplan-Meier survival curves for disease-free (A) and disease-specific (B) survival of 181 patients according to subcellular localization of maspin expression. C-only: Cytoplasmic-only, P: pancellular (nuclear and cytoplasmic), $N$-only: nuclear-only.

adenocarcinoma excluding AIS and MIA. To our knowledge, there is only a single report focusing on the subcellular localization of maspin expression in lung adenocarcinoma in which a tight association of nuclear expression of maspin with the lepidic growth pattern and a strong association of combined nuclear and cytoplasmic (pancellular) expression of maspin with invasion were observed (18). In the present study, the pancellular expression of maspin was not correlated with any clinicopathological factors, including histological subtype and the size of invasion. The main reason for this discrepancy may be the difference in antibody used as described previously (19). Using an in vivo model system, Goulet et al. (20) reported that the nuclear localization of maspin was required for its tumor- and metastasis-suppressor functions and that tumor cells expressing nucleus-excluded, cytoplasmic-only maspin were more metastatic than controls. Goulet et al. thus speculated that the cytoplasmic-only (but not pancellular) expression of maspin might correlate with an aggressive phenotype. Our present finding that the cytoplasmic-only (but not pancellular) expression of maspin was a predictor for unfavorable prognosis may support these experimental findings. However, our results failed to demonstrate that the cytoplasmic expression of maspin was an independent prognostic factor for DFS and DSS according to the multivariate analysis. The main reason for this failure may be the relatively small number of cases and the relatively short follow-up period in our study. 
Table III. Univariate analysis of various prognostic factors in 181 patients with pathological stage IA lung adenocarcinoma according to the eighth edition of the TNM classification.

\begin{tabular}{|c|c|c|c|c|c|c|c|}
\hline \multirow[t]{2}{*}{ Prognostic factor } & \multirow[t]{2}{*}{ Comparison } & \multicolumn{3}{|c|}{ Disease-free survival } & \multicolumn{3}{|c|}{ Disease-specific survival } \\
\hline & & HR & $95 \% \mathrm{CI}$ & $p$-Value & HR & $95 \% \mathrm{CI}$ & $p$-Value \\
\hline Age & $\geq 70$ vs. $<70$ Years & 1.945 & $1.070-3.538$ & 0.029 & 1.740 & $0.507-5.969$ & 0.378 \\
\hline Gender & Male $v s$. female & 1.278 & $0.732-2.229$ & 0.388 & 1.319 & $0.388-4.486$ & 0.658 \\
\hline Smoking history & Ever $v s$. never & 1.708 & $0.974-2.996$ & 0.062 & 1.446 & $0.441-4.743$ & 0.543 \\
\hline Surgery & Segmentectomy vs. lobectomy & 1.365 & $0.581-3.206$ & 0.475 & 2.175 & $0.520-7.405$ & 0.320 \\
\hline T Classification & pT1c vs. pT1a+1b & 1.869 & $1.066-3.277$ & 0.029 & 2.032 & $0.620-6.658$ & 0.242 \\
\hline Lymphatic invasion & Present $v s$. absent & 2.796 & $1.577-4.959$ & $<0.001$ & 5.044 & $1.538-16.540$ & 0.008 \\
\hline Cytoplasmic-only maspin expression & Positive $v s$. negative & 1.848 & $1.037-3.294$ & 0.037 & 3.323 & $1.011-10.916$ & 0.048 \\
\hline
\end{tabular}

HR, Hazard ratio; CI, Confidence interval.

Table IV. Multivariate analysis of various prognostic factors in 181 patients with pathological stage IA lung adenoca)rcinoma according to the eighth edition of the TNM classification.

\begin{tabular}{|c|c|c|c|c|c|c|c|}
\hline \multirow[t]{2}{*}{ Prognostic factor } & \multirow[t]{2}{*}{ Comparison } & \multicolumn{3}{|c|}{ Disease-free survival } & \multicolumn{3}{|c|}{ Disease-specific survival } \\
\hline & & HR & $95 \% \mathrm{CI}$ & $p$-Value & HR & $95 \% \mathrm{CI}$ & $p$-Value \\
\hline Age & $\geq 70$ vs. $<70$ Years & 2.163 & $1.184-3.950$ & 0.012 & 2.021 & $0.575-7.103$ & 0.272 \\
\hline $\mathrm{T}$ Classification & pT1c vs. pT1a+1b & 1.483 & $0.832-2.643$ & 0.182 & 1.213 & $0.341-4.316$ & 0.765 \\
\hline Lymphatic invasion & Present $v s$. absent & 3.055 & $1.716-5.440$ & $<0.001$ & 5.044 & $1.538-16.540$ & 0.008 \\
\hline Cytoplasmic-only maspin expression & Positive $v s$. negative & 1.306 & $0.705-2.422$ & 0.396 & 2.584 & $0.770-8.673$ & 0.124 \\
\hline
\end{tabular}

HR, Hazard ratio; CI, Confidence interval.

In conclusion, we demonstrated, for the first time, that the cytoplasmic-only (but not pancellular or nuclear-only) expression of maspin was a predictor of shorter DFS and DSS in patients with p-stage IA lung adenocarcinoma categorized by the new TNM classification. Due to the recent improvements in imaging techniques, stage I lung adenocarcinoma has been detected more often than in the past; it is, therefore, important to identify markers for stratifying patients with a small tumor but of aggressive phenotype so that appropriate treatment strategies can be selected as early as possible. Although further studies with larger series of patients and longer follow-up periods are needed, our findings suggest that the immunohistochemical analysis of maspin could be useful for the prediction of an aggressive tumor phenotype in patients with p-stage IA lung adenocarcinoma.

\section{Conflicts of Interest}

The Authors confirm that there are no conflicts of interest in regard to this study.

\section{Acknowledgements}

The Authors are grateful to Kazuo Yashima and Kazuko Fukushima for their excellent technical assistance with the processing of the pathological specimens.

\section{References}

1 Travis WD, Brambilla E, Burke AP, Marx A and Nicholson AG: WHO classification of tumours of the lung, pleura, thymus and heart, Fourth edition. IARC, Lyon, 2015.

2 Ujiie H, Kadota K, Chaft JE, Buitrago D, Sima CS, Lee MC, Huang J, Travis WD, Rizk NP, Rudin CM, Jones DR and Adusumilli PS: Solid predominant histologic subtype in resected stage I lung adenocarcinoma is an independent predictor of early, extrathoracic, multisite recurrence and poor postrecurrence survival. J Clin Oncol 33: 2877-2884, 2015.

3 Hung JJ, Jeng WJ, Chou TY, Hsu WH, Wu KJ, Huang BS and Wu YC: Prognostic value of the new International Association for the Study of Lung Cancer/American Thoracic Society/European Respiratory Society lung adenocarcinoma classification on death and recurrence in completely resected stage I lung adenocarcinoma. Ann Surgery 258: 1079-1086, 2013.

4 Kubouchi Y, Kidokoro Y, Ohno T, Yurugi Y, Wakahara M, Haruki $\mathrm{T}$ and Nakamura $\mathrm{H}$ : Prognostic factors for post recurrence survival in resected pathological stage I non-small cell lung cancer. Yonago Acta Medica 60: 213-219, 2017.

5 Brierley JD, Gospodarowicz MK and Wittekind C (eds.): TNM Classification of Malignant Tumours, 8th ed. Wiley-Blackwell, Oxford, 2017.

6 Goldstraw P, Chansky K, Crowley J, Rami-Port R, Asamura H, Eberhardt WEE, Nicholson AG, Groome P, Mitchell A and Bolejack V: The IASLC Lung Cancer Staging Project: Proposals for revision of the TNM stage groupings in the forthcoming 
(eight) edition of the TNM Classification for Lung Cancer. J Thorac Oncol 11: 39-51, 2016.

7 Zou Z, Anisowicz A, Hendrix MJ, Thor A, Neveu M, Sheng S, Rafidi K, Seftor E and Sagar R: Maspin, a serpin with tumorsuppressant activity in human mammary epithelial cells. Science 263: 526-529, 1994.

8 Bodenstine TM, Seftor RE, Khalkhali-Ellis Z, Seftor EA, Pemberton PA and Hendrix MJC: Maspin: molecular mechanisms and therapeutic implications. Cancer Metastasis Rev 31: 529-551, 2012.

9 Hirai K, Koizumi K, Haraguchi S, Hirata T, Mikami I, Fukushima M, Yamagishi S, Kawashima T, Okada D, Shimizu $\mathrm{K}$ and Kawamoto M: Prognostic significance of the tumor suppressor gene maspin in non-small cell lung cancer. Ann Thorac Surg 79: 248-253, 2005.

10 Nakagawa M, Katakura H, Adachi M, Takenaka K, Yanagihara $\mathrm{K}$, Otake $\mathrm{Y}$, Wada $\mathrm{H}$ and Tanaka F: Maspin expression and its clinical significance in non-small cell lung cancer. Ann Surg Oncol 13: 1517-1523, 2006.

11 Takanami I, Abiko T and Koizumi S: Expression of maspin in non-small-cell lung cancer: correlation with clinical features. Clin Lung Cancer 9: 361-366, 2008.

12 Woenckhaus M, Bubendorf L, Dalquen P, Foerster J, Blaszyk H, Mirlacher M, Soler M, Dietmaier W, Sauter G, Hartmann A and Wild PJ: Nuclear and cytoplasmic maspin expression in primary non-small cell lung cancer. J Clin Pathol 60: 483-486, 2007.

13 Lonardo F, Li X, Siddiq F, Singh R, Al-abbadi M, Pass HI and Sheng S: Maspin nuclear localization is linked to favorable morphological features in pulmonary adenocarcinoma. Lung Cancer 51: 31-39, 2006.

14 Frey A, Soubani AO, Adam AK, Sheng S, Pass HI and Lonardo F: Nuclear, compared with combined nuclear and cytoplasmic expression of maspin, is linked in lung adenocarcinoma to reduced VEGF-A levels and in stage I, improved survival. Histopathology 54: 590-597, 2009.
15 Takagi Y, Matsuoka Y, Shiomi T, Nosaka K, Takeda C, Haruki T, Araki K, Taniguchi Y, Nakamura $\mathrm{H}$ and Umekita $\mathrm{Y}$ : Cytoplasmic maspin expression is a predictor of poor prognosis in patients with lung adenocarcinoma measuring $<3 \mathrm{~cm}$. Histopathology 66: 732-739, 2015.

16 Murakami S, Ito H, Tsubokawa N, Mimae T, Sasada S, Yoshiya T, Miyata Y, Yokose T, Okada M and Nakayama H: Prognostic value of the new IASLC/ATS/ERS classification of clinical stage IA lung adenocarcinoma. Lung Cancer 90: 199-204, 2015.

17 Smith SL, Watson SG, Ratschiller D, Gugger M, Betticher DC and Heighway J: Maspin - the most commonly-expressed gene of the 18q21.3 serpin cluster in lung cancer - is strongly expressed in preneoplastic bronchial lesions: Oncogene 22: 8677-8687, 2003.

18 Lonardo F, Guan H, Dzinic S and Sheng S: Maspin expression patterns differ in the invasive versus lepidic growth pattern of pulmonary adenocarcinoma. Histopathology 65: 757-763, 2014.

19 Wakahara M, Sakabe T, Kubouchi Y, Hosoya K, Hiooka Y, Yurugi Y, Nosaka K, Shiomi T, Nakamura H and Umekita Y: Subcellular localization of maspin correlates with histone deacetylase 1 expression in human breast cancer. Anticancer Res 37: 5071-5077, 2017.

20 Goulet B, Chan G, Chambers AF and Lewis JD: An emerging role for the nuclear localization of maspin in the suppression of tumor progression and metastasis. Biochem Cell Biol 90: 22-38, 2012.
Received February 15, 2018

Revised March 6, 2018

Accepted March 8, 2018 